\title{
Minocycline inhibits PARP-1 expression and decreases apoptosis in diabetic retinopathy
}

\author{
YING WU ${ }^{1}$, YONGDONG CHEN ${ }^{2}$, QIANG WU ${ }^{2}$, LILI JIA ${ }^{2}$ and XINHUA DU ${ }^{2}$ \\ ${ }^{1}$ Department of Ultrasound, Shanghai First People's Hospital, School of Medicine, Shanghai Jiaotong University, \\ Shanghai 200080; ${ }^{2}$ Department of Ophthalmology, Shanghai Jiaotong University Affiliated Sixth People's Hospital, \\ Shanghai 200233, P.R. China
}

Received May 29, 2014; Accepted June 3, 2015

DOI: $10.3892 / \mathrm{mmr} .2015 .4064$

\begin{abstract}
The present study aimed to investigate the mechanism underlying the effects of minocycline on diabetic retinopathy-associated cellular apoptosis. A total of 40 Sprague Dawley (SD) rats were used as a diabetic retinopathy model following injection with streptozotocin. Among the 34 rats in which the diabetes model was successfully established, 24 rats were divided into two experimental groups: I and II (T1 and $\mathrm{T} 2$, respectively), and orally administered with various doses of minocycline. The remaining 10 rats served as the diabetic retinopathy control group. An additional group of 10 healthy SD rats with comparable weight served as normal controls. The rats in T1 and T2 groups were treated daily for eight consecutive weeks with minocycline at a dose of $2.5 \mathrm{mg} / \mathrm{kg}$ and $5 \mathrm{mg} / \mathrm{kg}$, respectively. The mRNA expression levels of poly (ADP-ribose) polymerase-1 (PARP-1) were subsequently measured by reverse transcription-quantitative polymerase chain reaction, and the protein expression levels of poly-ADP-ribose were measured by western blot analysis and immunohistochemistry. Retinal morphology was observed following hematoxylin and eosin staining, and retinal cell apoptosis was measured by terminal deoxynucleotidyl transferase dUTP nick end labeling and caspase-3 activity assays. The amplitudes of the electroretinogram (ERG) b-wave and oscillary potentials (OPs) were measured using visual electrophysiology, and compared among the four groups. The results of the present study demonstrated that in the diabetic rats, retinal PARP-1 gene expression was markedly upregulated, the number of apoptotic cells and the activity levels of caspase-3 were increased, and the amplitude of the ERG b-wave and the OPs were markedly lower as compared with the normal rats. Following treatment with minocycline, the abnormal expression of PARP-1 in the retina was inhibited,
\end{abstract}

Correspondence to: Dr Yongdong Chen, Department of Ophthalmology, Shanghai Jiaotong University Affiliated Sixth People's Hospital, 600 Yishan Road, Shanghai 200233, P.R. China E-mail: drchenyd@163.com

Key words: diabetic retinopathy, minocycline, apoptosis, poly (ADP-ribose) polymerase-1, electroretinogram and cellular apoptosis was decreased. In conclusion, the results of the present study suggest that PARP-1 is involved in the development of diabetic retinopathy, and minocycline is able to inhibit PARP-1 expression and decrease cellular apoptosis, suggesting that minocycline may prove to be a promising drug for the treatment of diabetic retinopathy.

\section{Introduction}

Apoptosis is one of the most important mechanisms underlying diabetic retinopathy. It is well-established that diabetes leads to a loss of vascular cells in the retina, as determined by retinal treatment with trypsin (1). Previous studies have demonstrated that diabetes increases the levels of neuronal apoptosis, specifically within the inner retina where the retinal ganglion cells are located, as determined by terminal deoxynucleotidyl transferase dUTP nick end labeling (TUNEL) assays of the histologic sections of streptozotocin (STZ)-diabetic rat retinas, and post-mortem human retinas $(2,3)$. In addition, neural apoptosis is an important event that occurs prior to vascular apoptosis, with the rate of neural apoptosis remaining constant throughout the duration of diabetes (4).

Poly (ADP-ribose) polymerase (PARP) is a nuclear enzyme that is involved in the cellular response to DNA damage (5). DNA breaks are believed to be triggers for the activation of PARP. When DNA damage is mild, PARP facilitates cell survival, whereas when DNA damage is severe, PARP activation induces cellular energetic disturbances, leading to cellular dysfunction or death (6-8). In the early stages of diabetic retinopathy, peroxynitrite enters the nucleus where it causes breaks in the DNA strand, thereby activating PARP (9). The abnormal activation of PARP caused by oxidative stress results in cell apoptosis, initiation of nuclear factor $(\mathrm{NF})-\kappa \mathrm{B}$, and release of inflammatory mediators (10), which are important mechanisms underlying the occurrence and development of diabetic retinopathy (11).

The therapeutic effects of minocycline have been confirmed in numerous studies; however the mechanism underlying these therapeutic effects has yet to be fully elucidated $(12,13)$. It has been suggested that minocycline may be associated with the inhibition of proinflammatory cytokine expression and glial cell activation $(14,15)$. A previous study demonstrated that minocycline is able to inhibit the abnormal expression 
of caspase-3 and PARP, and has a neuroprotective role in light-induced retinal injury (16). The present study aimed to investigate the expression of PARP-1 and cell apoptosis in the retinal tissue samples of diabetic rats, and to evaluate the effects of minocycline on PARP-1 expression and cell apoptosis, in order to elucidate the possible mechanisms underlying these effects.

\section{Materials and methods}

Reagents and kits. DNase I, minocycline, STZ and TRIzol ${ }^{\circledR}$ was obtained from Invitrogen Life Technologies (Carlsbad, CA, USA). RevertAid First Strand cDNA Synthesis kit was purchased from Fermentas AB (Vilnius, Lithuania). The VECTASTAIN $^{\circledR}$ ABC-Horseradish Peroxidase (HRP) kit was purchased from Vector Laboratories, Inc. (Burlingame, CA, USA). The primary mouse monoclonal anti-PAR antibody was purchased from Abcam (Cambridge, UK; cat. no. ab14459). The goat anti-mouse immunoglobulin G-HRP antibody was purchased from Santa Cruz Biotechnology, Inc. (Dallas, TX, USA; cat.no. sc-2005). The Enhanced Chemiluminescence (ECL) kit was purchased from GE Healthcare Life Sciences (Chalfont, UK). DEVD-AFC was purchased from BioVision, Inc.(Milpitas, CA, USA). The Bicinchoninic Acid(BCA) kit was purchased from Beyotime Institute of Biotechnology (Jiangsu, China). The FragEL ${ }^{\text {TM }}$ DNA Fragmentation Detection kit was purchased from Merck Millipore (Darmstadt, Germany). The ABI PRISM ${ }^{\circledR} 7500$ Fast Sequence Detection system was purchased from Applied Biosystems Life Technologies (Foster City, CA, USA). The Tecan Spectrafluor Plus plate reader was purchased from Nonlinear USA, Inc. (Durham, NC, USA). The UTAS-E2000 electrophysiological recording and analyzing system was purchased from LKC Technologies, Inc. (Gaithersburg, MD, USA).

Animals and groups. The protocols for animal care and use adhered to the principles set forth in the ARVO Statement for the Use of Animals in Ophthalmic and Vision Research (http://www. arvo.org/About_ARVO/Policies/Statement_for_the_Use_of_ Animals_in_Ophthalmic_and_Visual_Research/). A total of 50 male Sprague Dawley (SD) rats (age, 6 weeks; weight, 200-250 g) were purchased from the Shanghai Laboratory Animal Center (Shanghai, China). The rats were housed at a temperature of $20-24^{\circ} \mathrm{C}$, at $55-70 \%$ humidity under a $12-\mathrm{h}$ light/dark cycle. The rats were randomly divided into two groups: A total of 10 rats were assigned to the normal control (CON) group, and 40 rats to the diabetic model (DM) group. The diabetic rat model was established as follows: After food and water deprivation for $12 \mathrm{~h}$, the rats were intraperitoneally injected with $60 \mathrm{mg} / \mathrm{kg} \mathrm{STZ}$ solution (STZ dissolved in $0.1 \mathrm{~mol} / 1$ citric acid sodium citrate buffer, $\mathrm{pH} 4.5$ ). Two days following STZ injection, blood samples were obtained from the rat tail vein and used to measure the levels of fasting blood glucose using a One Touch Horizon ${ }^{\mathrm{TM}}$ blood glucose meter [Johnson \& Johnson Medical (China) Ltd., Shanghai, China]. The rats with blood glucose levels $>16.7 \mathrm{mM}$ were considered successful models of diabetes. The control rats received intraperitoneal injection of an equal volume of $0.1 \mathrm{~mol} / \mathrm{l}$ citric acid-sodium citrate buffer at $\mathrm{pH} 4.5$. Blood glucose levels were monitored weekly.
Among the 40 rats treated with STZ, the diabetic model was successfully established in 34. Two weeks following the establishment of the model, 24 of the 34 rats were randomly divided into two groups $(\mathrm{n}=12)$, and orally treated with either $2.5 \mathrm{mg} / \mathrm{kg}$ minocycline (T1 group), or $5 \mathrm{mg} / \mathrm{kg}$ minocycline (T2 group). The treatment was given once a day for eight consecutive weeks. The other 10 diabetic model rats served as diabetic controls and were treated with saline. The remaining 10 normal rats served as the normal control group.

Following the establishment of the STZ-induced diabetic rat model, rat distal blood glucose and body weight were regularly monitored and recorded. A slit lamp and ophthalmoscope were used to observe any changes in the anterior or posterior segments of the rat eyes. If any changes occurred, images of the rat eyes were captured. Following 10 consecutive weeks, an electroretinogram (ERG) examination was performed. A total of 10 rats from each group were subsequently sacrificed by anesthetic overdose (dose, $5 \mathrm{ml}$ ) with $2 \%$ pentobarbital. The eyes of the rats were removed, and 8 of the eyes were placed in $10 \%$ formaldehyde solution for hematoxylin and eosin (HE) staining, and paraffin fixation. The remaining 12 eyes were flushed with saline, prior to the front sections being removed and the retina isolated. The retinal tissue samples were cryopreserved in liquid nitrogen and used for reverse transcription-quantitative polymerase chain reaction (RT-qPCR), western blotting, and caspase-3 activity measurements. The present study was approved by the Shanghai Jiaotong University Affiliated Sixth People's Hospital Animal Welfare Ethics Committee (Shanghai, China).

Quantification of the MRNA expression levels of PARP-1 by $R T-q P C R$. Total RNA was extracted using the TRIzol ${ }^{\circledR}$ method. Any contaminating DNA was removed from the RNA preparations by DNase I treatment, and $4 \mu \mathrm{g}$ RNA was reverse transcribed using the First Strand cDNA Synthesis kit. RT-qPCR was performed using an ABI PRISM ${ }^{\circledR} 7500$ Fast Sequence Detection system with a $S Y B{ }^{\circledR}$ green fluorescent label [Takara Biotechnology (Dalian) Co., Ltd., Dalian, China]. The primer sequences used in the RT-qPCR assays for PARP-1 and $\beta$-actin were as follows: PARP-1 forward, 5'-CGGCACGAGAGGGAGGATGG-3', and reverse, 5'-TGTCAGGCTGCCGGATGGAGT-3'; and $\beta$-actin forward, 5'-TGTGATGGTGGGTATGGGTCAGAAG-3', and reverse, 5'-TCACGGTTGGCCTTAGGGTTCAGAG-3' (obtained from Sangon Biotech, Shanghai, China). The reaction was conducted using a 20- $\mu$ l RT-qPCR solution containing $0.4 \mu \mathrm{l}$ primer, 10.0 $\mu \mathrm{l}$ SYBR Premix Ex Taq (2x), 1.0 $\mu 1$ DNA template, and diethylpyrocarbonate- $\mathrm{ddH}_{2} \mathrm{O}$, with the following cycling parameters: $95^{\circ} \mathrm{C}$ for $30 \mathrm{sec}, 95^{\circ} \mathrm{C}$ for $5 \mathrm{sec}$, and $60^{\circ} \mathrm{C}$ for $30 \mathrm{sec}$ for 40 cycles. In each sample $\beta$-actin served as an internal reference to standardize the results by eliminating variations in mRNA quantity.

Western blot analysis. Retinal proteins were extracted using protein cleavage buffer and protease inhibitors $(30 \mathrm{mM}$ Tris $\mathrm{pH} 7.5,150 \mathrm{mM} \mathrm{NaCl}, 1 \mathrm{mM}$ phenylmethylsulfonyl fluoride, $1 \mathrm{mM} \mathrm{Na} \mathrm{VO}_{4}, 1 \%$ Nonidet P-40, and $10 \%$ glycerol) and the amount of total protein was quantified using the BCA kit. Samples with equal amounts of protein content were separated by $10 \%$ SDS-PAGE and $20 \mu \mathrm{g}$ protein was transferred 
to polyvinylidene fluoride membranes (Beyotime Institute of Biotechnology Co., Ltd., Jiangsu, China). The membranes were blocked with 5\% non-fat milk at room temperature for $2 \mathrm{~h}$, and then incubated with the appropriate primary antibody $(1: 1,000)$ overnight at $4^{\circ} \mathrm{C}$. Following three washes with Tris-buffered saline (TBS) Tween ${ }^{\circledR} 20$, the membranes were incubated with HRP-conjugated secondary antibody $(1: 5,000)$ at room temperature for $1 \mathrm{~h}$. The bands were visualized using an ECL reagent. The band intensities were subsequently quantified using Quantity One software (version 4.62; Bio-Rad Laboratories, Inc., Hercules, CA, USA) and normalized to $\beta$-actin as an internal standard.

Immunohistochemistry (IHC). The rat eyes were embedded in paraffin wax and sectioned along the axis oculi. Two of the sections were used for HE staining, and the others for IHC. IHC was performed using the VECTASTAIN ${ }^{\circledR}$ ABC-HRP kit. Phosphate-buffered saline (PBS) was substituted for the primary antigen in order to serve as a negative control, and a confirmed positive section was used as a positive control. Briefly, sections of the eyes were dewaxed and rehydrated in TBS prior to being boiled in $0.01 \mathrm{~mol} / \mathrm{l}$ sodium citrate buffer ( $\mathrm{pH}$ 6.0) for $15 \mathrm{~min}$. Endogenous peroxidase activity was suppressed with $0.3 \%$ hydrogen peroxide. The samples were then blocked with $10 \%$ normal goat serum (Beyotime Institute of Biotechnology Co., Ltd.) for $30 \mathrm{~min}$. The primary PAR antibodies (1:100) were added to the slides and incubated overnight at $37^{\circ} \mathrm{C}$ in a humidified atmosphere. PBS was added to substitute the primary antibody and act as a negative control. Following washing, the appropriate biotinylated secondary antibody was added to the sections at a 1:200 dilution, and incubated for $30 \mathrm{~min}$ at $37^{\circ} \mathrm{C}$, prior to being washed in TBS. The biotinylated enzyme complex was then added to the section for $30 \mathrm{~min}$ at $37^{\circ} \mathrm{C}$ in humidified atmosphere. Detection was conducted using a diaminobenzidine kit (Beyotime Institute of Biotechnology Co., Ltd.).

TUNEL assay. The rat eyes were sectioned along the vertical meridian in order to include the full length retina passing through the optic nerve head and the superior and inferior regions of the eye. Apoptosis was detected by TUNEL assay using the FragEL ${ }^{\mathrm{TM}}$ DNA Fragmentation Detection kit, according to the manufacturer's instructions. The neuronal apoptosis index in the retina was calculated as follows: Apoptotic index $=$ apoptotic cells/total cell number within the same field $x$ 100. The TUNEL-positive cells were quantified for the ganglion cell layers (GCL) and inner nuclear layers (INL), respectively, in order to distinguish the cell types affected, due to the fact that TUNEL-positive cells were predominantly located in the inner retina. For each sample, three sections were selected and the cells were counted from each section within five independent fields. The average overall index from six specimens of each group was considered as the apoptotic index of this group.

Measurement of caspase-3 activity. The amount of total protein was quantified using the BCA kit, and equal amounts of retinal protein extracts $(15 \mu \mathrm{g})$ were incubated in lysate buffer containing the fluorogenic DEVD-AFC substrate $(2.5 \mu \mathrm{M})$ at a total volume of $100 \mu \mathrm{l}$, at $37^{\circ} \mathrm{C}$ for $1 \mathrm{~h}$. The fluorescence signal emitted by cleavage of the substrate was quantified by a fluorescence plate reader.

Electroretinogram (ERG). Following dark adaptation for $30 \mathrm{~min}$, the rats were anesthetized by intraperitoneal injection with $70 \mathrm{mg} / \mathrm{kg}$ ketamine hydrochloride (Sangon Biotech). A total of $1 \%$ tropicamide eye drops were then used to dilate the rat pupils. The corneal surfaces were anesthetized using 4\% hydrochloric acid Oxybuprocaine eye drops (Santen Pharmaceutical Co., Ltd., Shanghai, China). The rats were then placed onto a hot plate which was heated to $\sim 37.5^{\circ} \mathrm{C}$, in order to reduce the impact of anesthesia on the electrophysiological test. The reference electrode was subcutaneously placed on the foreheads of the rats, and the ground electrode was placed on their right ears. A silver chloride ring-recording electrode was placed on the surface of the corneas of the rats, and coated with Carbomer Eye gel (Bausch \& Lomb, Rochester, NY, USA). The non-experimental eye was covered with a black cloth. A UTAS-E 2000 electrophysiological recording and analyzing system was used to examine the rat eye with the following parameters: The full-field stroboscopic white light stimulus distance was $30 \mathrm{~cm}$, the pass band was $75-300 \mathrm{~Hz}$, and the stimulus intensity long-flash $\left(\mathrm{cd} . \mathrm{s} / \mathrm{cm}^{2}\right)$ had a duration of $10 \mu \mathrm{sec}$, with every sub-interval lasting $1 \mathrm{sec}$. The mixed maximum b-wave amplitude and the sum of the oscillatory potential (OP) amplitudes during dark adaptation were recorded. The mean values were calculated for both eyes.

Statistical analysis. The experimental data are expressed as the mean \pm standard deviation, and statistical analyses were performed using the SAS6.12 statistical package (SAS Institute Inc, NC, USA). Following testing of normality and homogeneity of variance, the expression of PARP-1, activity of caspase-3, apoptosis index, and ERG amplitude among the four groups were analyzed by one-way analysis of variance, and the statistical differences between any two groups were analyzed by Student Newman Keuls or Dunnett tests using two-tailed P-values. $\mathrm{P}<0.05$ was considered to indicate a statistically significant difference.

\section{Results}

Blood glucose and body weight. The diabetic model was successfully established in 34 rats. Blood glucose levels ranged between 18.3 and $31.8 \mathrm{mmol} / \mathrm{l}$, with an average of $26.09 \pm 3.50 \mathrm{mmol} / 1$. The average body weight was $205.42 \pm 6.97 \mathrm{mg}$. The diabetic rats gradually exhibited polydipsia, polyphagia, and polyuria, progressively lost weight, and developed an arched posture. The rats were then randomly divided into a diabetic animal model group (DM), $2.5 \mathrm{mg} / \mathrm{kg} / \mathrm{d}$ minocycline treatment group (T1), and $5 \mathrm{mg} / \mathrm{kg} / \mathrm{d}$ minocycline treatment group (T2). The body weights at various time points in the DM, T1, and T2 groups showed no significant difference, but were markedly lower as compared with the normal control group $(\mathrm{CON})(\mathrm{P}<0.05)$. Blood glucose and body weight at various time points are shown in Table I.

Expression levels of PARP-1 in the retinal tissue samples of the DM, T1, T2, and CON groups. The mRNA expression levels of PARP-1 in the retinal tissue samples of the CON 
Table I. Rat blood glucose and body weight at various time points. The body weight of the DM, T1, and T2 groups showed no statistically significant difference at 0 weeks, but was significantly lower, as compared with the CON group, at 4-8 weeks $(\mathrm{P}<0.05)$.

\begin{tabular}{lccc}
\hline Group & Number & Blood glucose $(\mathrm{mmol} / \mathrm{l})$ & Body weight $(\mathrm{g})$ \\
\hline 0 weeks & & & \\
CON & 10 & $5.20 \pm 0.25$ & $205.68 \pm 7.12$ \\
DM & 10 & $27.77 \pm 2.04$ & $203.75 \pm 7.24$ \\
T1 & 12 & $26.29 \pm 3.91$ & $202.65 \pm 5.72$ \\
T2 & 12 & $24.22 \pm 3.59$ & $209.85 \pm 6.15$ \\
4 weeks & & & \\
CON & 10 & $5.14 \pm 0.12$ & $450.11 \pm 26.30$ \\
DM & 10 & $29.17 \pm 3.05$ & $244.02 \pm 7.06^{\mathrm{a}}$ \\
T1 & 12 & $27.29 \pm 3.80$ & $242.43 \pm 6.22^{\mathrm{a}}$ \\
T2 & 12 & $28.12 \pm 4.40$ & $239.15 \pm 7.15^{\mathrm{a}}$ \\
8 weeks & & \\
CON & 10 & $5.31 \pm 0.34$ & $505.22 \pm 31.28$ \\
DM & 10 & $31.77 \pm 5.01$ & $252.70 \pm 5.42^{\mathrm{a}}$ \\
T1 & 12 & $29.28 \pm 4.98$ & $251.55 \pm 6.27^{\mathrm{a}}$ \\
T2 & 12 & $29.24 \pm 4.70$ & $249.21 \pm 6.33^{\mathrm{a}}$ \\
\hline
\end{tabular}

CON, normal control group; DM, diabetic retinopathy model group; T1, $2.5 \mathrm{mg} / \mathrm{kg}$ minocyclin treatment group; T2, $5 \mathrm{mg} / \mathrm{kg} \mathrm{minocycline}$ treatment group. The results are presented as the mean \pm standard deviation. ${ }^{a} \mathrm{P}<0.05$ vs. CON.

group, DM group, T1 group, and T2 group were 1.0, 2.28, 1.41 , and 1.20 , respectively (Fig. 1). The differences in the expression levels of PARP-1 between the DM group and the other three groups were found to be statistically significant ( $\mathrm{F}=12.56, \mathrm{P}=0.0021)$; however no statistically significant differences were observed between the CON and treatment groups. The mRNA expression levels of PARP-1 in the retinal tissue samples of the DM rats were significantly higher, as compared with the $\mathrm{CON}$ group. Following minocycline treatment, the abnormally elevated mRNA expression levels of PARP-1 in the retinal tissue samples of the DM rats were suppressed $(\mathrm{F}=12.56, \mathrm{P}=0.0021)$, as compared with the $\mathrm{DM}$ group. There was no statistically significant difference in the expression levels of PARP-1 between the T1 and T2 treatment groups $(\mathrm{P}>0.05)$.

Protein expression levels of PAR proteins in the retinal tissue samples of the DM, T1,T2, and CON groups. IHC showed that the protein expression levels of PAR in the retinal tissue samples of the DM rats were significantly higher, as compared with the CON group (Fig. 2). Positive PAR protein expression was observed in the retinal GCL) the INL, and the outer nuclear layer. Following treatment with minocycline, the expression levels of the PAR proteins were downregulated in the retinal tissue samples of the DM rats. The results of the western blot analysis demonstrated the presence of statistically significant differences in the expression levels of PAR proteins between the DM group and the other three groups $(\mathrm{F}=39.02, \mathrm{P}<0.01)$; however no statistically significant difference was observed between the treatment and CON groups.

Apoptosis in the retinal tissue samples of the DM, T1,T2, and CON groups. TUNEL imaging demonstrated that a

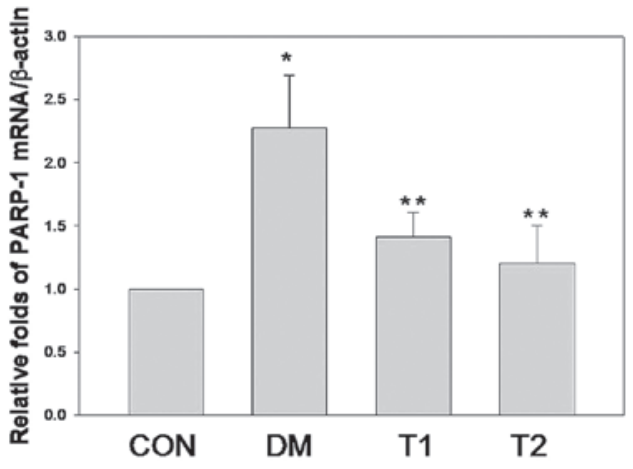

Figure 1. mRNA expression levels of poly (ADP-ribose) polymerase-1 (PARP-1) in the retinal tissue samples of the CON, DM, T1, and T2 groups. The mRNA expression levels of PARP-1 in the retinal tissue samples of the DM rats were significantly higher, as compared with the CON group. Following minocycline treatment, the abnormally elevated mRNA expression levels of PARP-1 in the retinal tissue samples of the DM rats were suppressed $(\mathrm{F}=12.56, \mathrm{P}=0.0021)$, as compared with the DM group. No statistically significant difference was observed in PARP-1 gene expression between the T1 and $\mathrm{T} 2$ treatment groups $(\mathrm{P}>0.05)$. ${ }^{*} \mathrm{P}<0.01$ vs. the other groups; ${ }^{* *} \mathrm{P}<0.05$ vs. CON. CON, normal control group; DM, diabetic retinopathy group; T1, $2.5 \mathrm{mg} / \mathrm{kg}$ minocycline treatment group; T2, $5 \mathrm{mg} / \mathrm{kg}$ minocycline treatment group.

small number of apoptotic cells were present in the rat retinal tissue samples of the CON group (Fig. 3). Following treatment for 10 weeks, the HE staining in the DM rats appeared normal, however the number of apoptotic cells had significantly increased, as compared with the CON group. The apoptotic cells were predominantly located in the GCL and the INL. The apoptotic index of the GCL in the four groups (CON, DM, T1 and T2) was $3.4 \pm 1.14,21.2 \pm 3.56$, $11.8 \pm 2.04$, and $10.8 \pm 1.30 \%$, respectively. The apoptotic index 


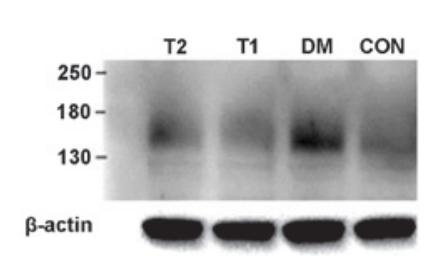

C

D
B

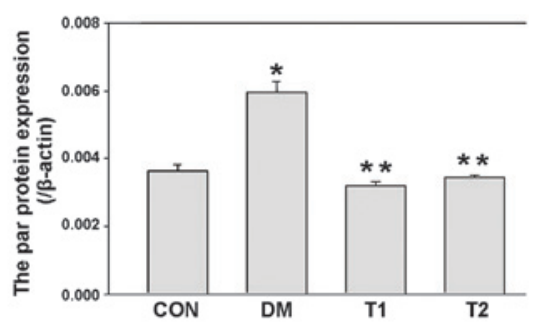

$\mathbf{E}$

$\mathbf{F}$

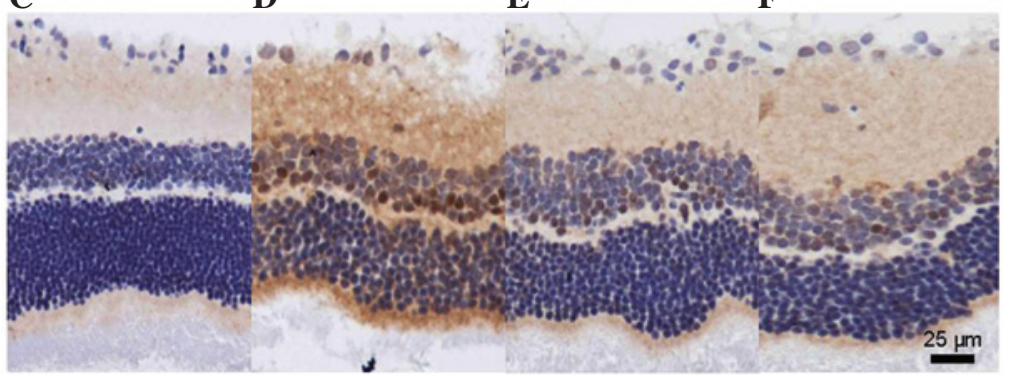

Figure 2. Expression levels of PAR proteins in the retinal tissue samples of DM rats were significantly higher, as comapred with the CON group. Following minocycline treatment, the expression levels of the PAR proteins were downregulated in the DM rats, as compared with the DM group. (A and B) Western blot analysis demonstrated a significant increase in the protein expression levels of the PAR proteins of the DM group, as compared with the other three groups, however no significant difference was observed between the CON, T1 and T2 groups ( $\mathrm{F}=39.02)$. " $\mathrm{P}<0.01$ vs. CON; * $\mathrm{P}<0.01$ vs. DM. (C) PAR expression was observed in the retinal ganglion cell layer, inner nuclear layer, and outer nuclear layer, as determined by immunohistochemistry. The PAR protein staining in the retinal tissue samples of the CON group was weakly positive. (D) Positive PAR expression increased significantly in the DM rats. (E and F) Following minocycline treatment, the expression levels of the PAR proteins were downregulated in the T1 and T2 groups. Scale bars, $50 \mu \mathrm{m}$. CON, normal control group; $\mathrm{DM}$, diabetic retinopathy group; T1, $2.5 \mathrm{mg} / \mathrm{kg}$ minocycline treatment group; T2, $5 \mathrm{mg} / \mathrm{kg}$ minocycline treatment group; PAR, poly (ADP-ribose).

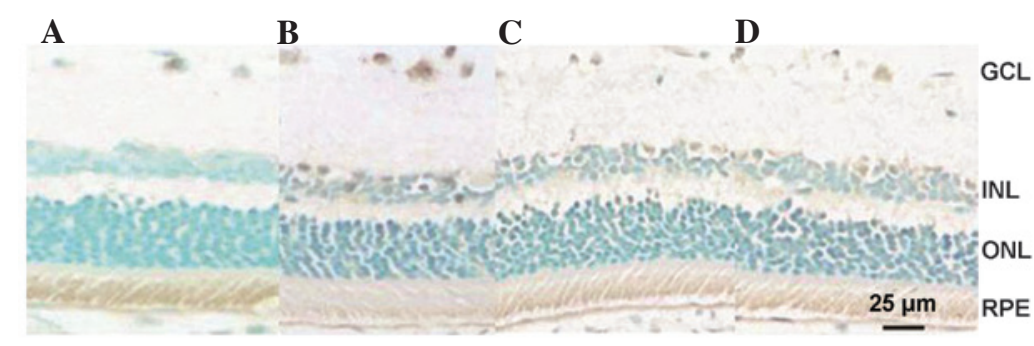

$\mathbf{E}$
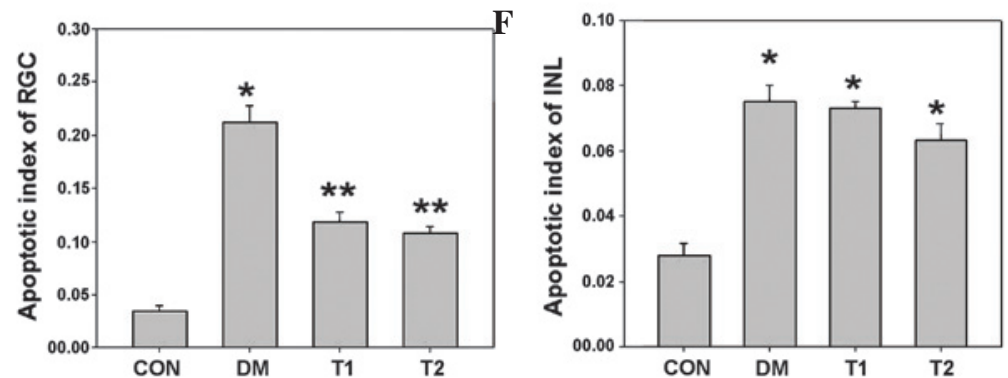

Figure 3. The number of apoptotic cells increased significantly following 10 weeks in the DM rats, and decreased following minocycline treatment. (A) A small number of apoptotic cells were present in the rat retinal tissue samples of the CON group. (B) The number of apoptotic cells increased significantly following 10 weeks in the DM rats. The apoptotic cells were located in the GCL, INL and ONL. (C and D) Following minocycline treatment, significantly less apoptotic cells were present in the retinal tissue samples of the DM rats in the T1 and T2 groups. (E) The apoptotic index in the GCL of the DM rats was significantly higher, as compared with the CON group. Following minocycline treatment, the elevated apoptotic index in the GCL of the retinal tissue samples of the DM rats was suppressed $\left(\mathrm{F}=53.57\right.$; ${ }^{\mathrm{P}} \mathrm{P}<0.01$ vs. $\left.\mathrm{CON}\right)$. No significant difference was observed between the GCL apoptotic index of the T1 and $\mathrm{T} 2$ treatment groups $($ (*) $>0.05)$. (F) Apoptotic index of the INL of the DM rats was significantly elevated, as compared with the CON group. Following minocycline treatment, the elevated apoptotic index in the INL of the retinal tissue samples of the DM rats was unchanged ( $\mathrm{F}=28.32 ;{ }^{*} \mathrm{P}<0.01$ vs. CON). No significant difference was observed between the INL apoptotic index of the T1 and T2 treatment groups ( $\mathrm{P}>0.05)$. (Scale bars, $25 \mu \mathrm{m})$. CON, control group; DM, diabetic retinopathy group; T1, $2.5 \mathrm{mg} / \mathrm{kg}$ minocycline treatment group; T2, $5 \mathrm{mg} / \mathrm{kg}$ minocycline treatment group; GCL, ganglion cell layer; ONL, outer nuclear layer; INL, inner nuclear layer.

of the INL was $2.8 \pm 0.84,7.5 \pm 1.12,7.3 \pm 0.45$, and $6.3 \pm 1.10 \%$, respectively. The apoptotic index of the GCL and INL in the DM rats were significantly higher, as compared with the
CON group $(\mathrm{F}=53.57, \mathrm{P}<0.01 ; \mathrm{F}=28.32, \mathrm{P}<0.01)$. Following minocycline treatment, the elevated apoptotic index of GCL in the retinal tissue samples of the DM rats was suppressed 


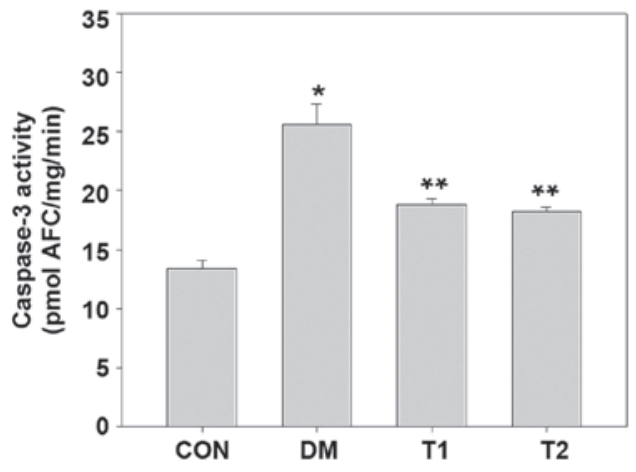

Figure 4. The activity levels of caspase-3 in the retinal tissue samples of the CON, DM, T1 and T2 groups. The activity levels of caspase- 3 were significantly elevated in the retinal tissue samples of the DM rats, as compared with the CON group. Following minocycline treatment, the elevated activity levels of caspase-3 in the retinal tissue samples of the DM rats was suppressed $\left(\mathrm{F}=27.24 ;{ }^{*} \mathrm{P}<0.01\right.$ vs. $\mathrm{CON}$ and ${ }^{* *} \mathrm{P}<0.01$ vs. $\left.\mathrm{DM}\right)$. No statistically significant difference in the activity levels of caspase- 3 was observed between the T1 and $\mathrm{T} 2$ treatment groups $(\mathrm{P}>0.05) \mathrm{CON}$, normal control group; DM, diabetic retinopathy group; T1, $2.5 \mathrm{mg} / \mathrm{kg}$ minocycline treatment group; T2, $5 \mathrm{mg} / \mathrm{kg}$ minocycline treatment group.

$(\mathrm{F}=53.57, \mathrm{P}<0.01)$, whereas the apoptotic index of the INL in the retinal tissue samples of the DM rats was unchanged $(\mathrm{P}>0.05)$, as compared with the $\mathrm{CON}$ group.

Caspase-3 activity in the retinal tissue samples of the DM, $T 1, T 2$, and CON groups. The activity levels of caspase-3 were significantly increased $(\sim 91 \%)$ in the retinal tissue samples of the DM group, as compared with the CON group. The activity levels of caspase- 3 decreased in the T1 and T2 treatment groups by $\sim 26.6$ and $28.9 \%$, respectively, as compared with the DM group. Following minocycline treatment, the elevated activity levels of caspase-3 in the retinal tissue samples of the DM rats was suppressed $(\mathrm{F}=27.24, \mathrm{P}<0.01)$. No statistically significant difference in the activity levels of caspase- 3 was observed between the T1 and T2 treatment groups $(\mathrm{P}>0.05)$.

Electroretinograms. The b-wave amplitudes of the CON group, DM group, and T1 and T2 treatment groups were $255.7 \pm 32.79,148.3 \pm 32.62,161.1 \pm 31.47$, and $197.1 \pm 33.19 \mu \mathrm{V}$, respectively (Fig. 4 and 5). The differences between all of the groups were statistically significant $(\mathrm{F}=32.52, \mathrm{P}<0.0001)$. The b-wave amplitude in the DM group was significantly lower, as compared with the CON, T1 and T2 groups. The b-wave amplitude and OPs in the $\mathrm{T} 1$ and $\mathrm{T} 2$ treatment groups were lower than in the CON group, but no statistically significant difference was observed between the T1 and T2 groups. The b-wave amplitude in the CON, DM, and T1 and T2 groups was $125.9 \pm 19.47,76.5 \pm 16.72,97.8 \pm 15.82$, and $101.0 \pm 10.45 \mu \mathrm{V}$ respectively. Statistically significant differences were present among all four groups. The OP amplitudes in the DM group were significantly lower than in the CON group $(\mathrm{F}=10.46$, $\mathrm{P}=0.0011$ ). Following treatment with minocycline, the OP amplitude in the $\mathrm{T} 1$ and $\mathrm{T} 2$ groups was significantly higher as compared with the DM group $(\mathrm{P}<0.01)$, and lower as compared with the CON group $(\mathrm{P}<0.05)$. The differences in OPs between the T1 and T2 groups was not statistically significant $(\mathrm{P}>0.05)$.
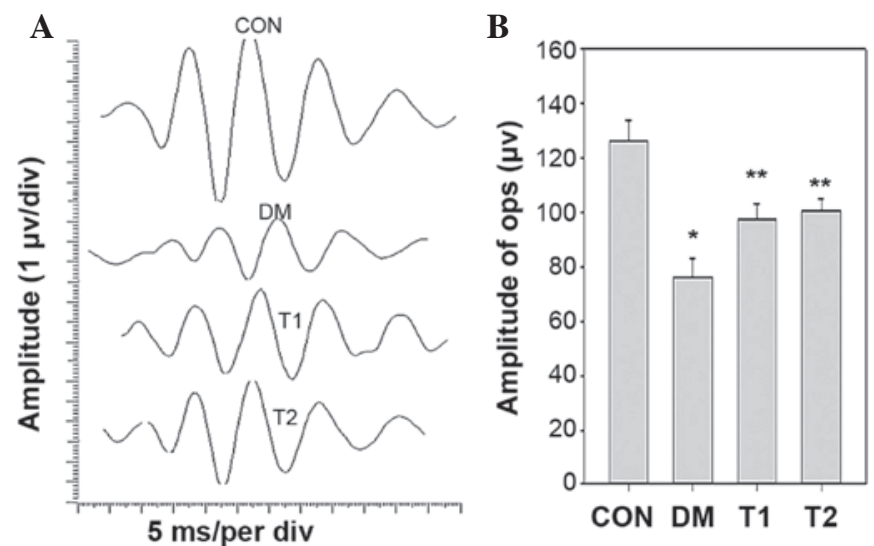

Figure 5. OPs of the retina in the CON, DM, T1 and T2 groups. (A) OPs of the rats in the CON, DM, T1 and T2 groups. (B) Amplitude analysis of the rat OPs in the CON, DM, T1 and T2 groups. The OP amplitudes in the DM group was significantly lower, as compared with the CON group $(\mathrm{F}=10.46 ; \mathrm{P}=0.0011) .{ }^{*} \mathrm{P}<0.01$ vs. $\mathrm{CON}$. Following minocycline treatment, the OP amplitude increased, but remained lower as compared with the CON group. No statistically significant difference was observed between the OP amplitudes of the T1 and T2 groups $\left({ }^{* *} \mathrm{P}>0.05\right)$. CON, normal control group; $\mathrm{DM}$, diabetic retinopathy group; $\mathrm{T} 1,2.5 \mathrm{mg} / \mathrm{kg}$ minocycline treatment group; $\mathrm{T} 2,5 \mathrm{mg} / \mathrm{kg}$ minocycline treatment group; OP, oscillary potential.
A

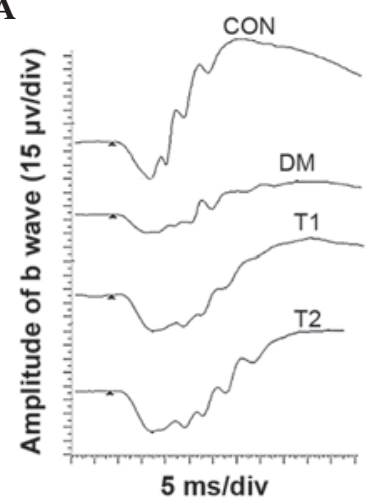

B

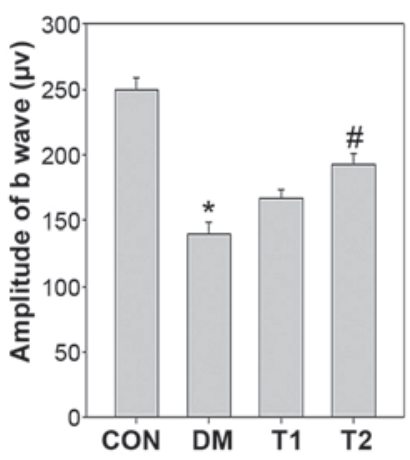

Figure 6. B-waves of the rats in the CON, DM, T1 and T2 groups, as demonstrated by ERG. (A) B-waves of the rats in the CON, DM, T1 and T2 groups. (B) Amplitude analysis of the ERG b-waves of the rats in all four groups. The b-wave amplitude in the DM group was significantly lower, as compared with the CON group $\left(\mathrm{F}=32.52 ;{ }^{*} \mathrm{P}<0.0001\right.$ vs. $\left.\mathrm{CON}\right)$, and following minocycline treatment, the b-wave amplitude increased, but remained lower than that of the CON group. The b-waves in the T2 groups were significantly greater, as compared with the T1 group ( ${ }^{\#} \mathrm{P}<0.001$ vs. T1). CON, normal control group; $\mathrm{DM}$, diabetic retinopathy group; $\mathrm{T} 1,2.5 \mathrm{mg} / \mathrm{kg}$ minocycline treatment group; $\mathrm{T} 2,5 \mathrm{mg} / \mathrm{kg}$ minocycline treatment group; ERG, electroretinogram.

\section{Discussion}

In the present study, a diabetic rat model was established by STZ intraperitoneal injection. The rats were subsequently treated with various doses of minocycline for 2 weeks. Following eight consecutive weeks, the retinal tissue samples of the DM rats expressed higher levels of PARP-1, as compared with the CON group, and retinal cell apoptosis also increased. The maximum potential b-wave and OP amplitudes decreased in the DM rats, as indicated by retinal ERG. Following minocycline treatment, the aberrant expression of PARP-1 in the retinal tissue samples was suppressed, the rate of cell apoptosis decreased, and the b-wave and OP amplitudes in 
ERG increased. These results indicated that abnormal PARP activation may have an important role in the mechanism that underlies the apoptosis that occurs during diabetic retinopathy, suggesting that minocycline may lower the levels of apoptosis via the inhibition of PARP. The present study demonstrated that the expression levels of PARP-1 genes and PAR proteins were significantly increased in the diabetic rats subjected to 10 weeks of modeling. Although no marked pathological changes were observed in the HE sections, IHC staining demonstrated that the expression levels of the PAR proteins were significantly increased in the retinal tissue samples of the DM rats, and these results were concordant with the western blot analysis. IHC demonstrated the presence of PAR protein expression in the inner retina. In addition, the apoptotic index and activity levels of caspase-3 were significantly increased in the retina of the diabetic rats, and the maximum potential b-wave and OP amplitudes decreased, as demonstrated by ERG. These results were concordant with the findings of previous studies (17-19). However, unlike previous studies, the present study included an investigation into the effects of orally administered minocycline, at $2.5 \mathrm{mg} / \mathrm{kg} / \mathrm{d}$ and $5 \mathrm{mg} / \mathrm{kg} / \mathrm{d}$, on PARP-1 expression and retinal cell apoptosis in diabetic rats. To our knowledge, the present study is the first to suggest that minocycline inhibits the aberrant expression of PARP in a dose-independent manner, and reduces the rate of retinal cell apoptosis in diabetic rats. Following minocycline treatment, the ERG b-waves and OP amplitudes were partially increased, with minocycline affecting ERG b-wave amplitudes in a dose-dependent manner, and OPs in a dose-independent manner.

The ERG is a useful method to investigate the mechanisms underlying retinal physiology and their alterations in disease. The b-waves of the ERG are associated with the function of Müller cells, on-bipolar cells, amacrine cells, and ganglion cells $(20,21)$. OPs are four to six wavelets in the ERG that are located on the rising phase of the b-wave (22), and were first shown to arise in the inner plexiform layer (23). A previous study suggested that OPs are generated by amacrine cells (24). Reduced OP and b-wave amplitudes are commonly observed in the early stages of disease prior to the onset of retinopathy. It is also well-accepted that ERG assesses retinal function, and provides an early warning of retinal abnormalities prior to any detectable visible alterations in diabetes (25). ERG abnormalities in the diabetic retina are not only due to apoptosis of retinal neurons, glial activation and glucose control are also involved in ERG abnormalities $(26,27)$. However, the present study demonstrated that the b-wave and OP amplitudes of diabetic SD rats were associated with the apoptosis of retinal neurons, which suggests that apoptosis is an important factor in early retinal dysfunction due to diabetes mellitus.

The mechanism underlying apoptosis in diabetic retinopathy has yet to be fully elucidated. Proven mechanisms include oxidative stress, abnormal activation of glial cells, and proinflammatory cytokine expression $(14,15,28,29)$. Previous studies have shown that PARP activation has an important role in diabetic retinopathy apoptosis $(17,30,31)$. There are two possible mechanisms by which PARP activation may lead to apoptosis: Consumption of cellular $\mathrm{NAD}^{+}$resulting in cell energy failure and apoptosis, or regulation of $\mathrm{NF}-\kappa \mathrm{B}$ gene expression that upregulates inflammatory factors, leading to cell apoptosis (10,32-35). In the present study, apoptosis was associated with the upregulation of PARP-1 in the retinal tissue samples of diabetic rats. Treatment with minocycline inhibited PARP-1 expression and decreased apoptosis. These results are concordant with those of a previous study (32), which demonstrated that the activity levels of PARP were significantly increased in the retinas of diabetic rats. PARP activation led to cell apoptosis and the release of inflammatory mediators, such as adhesion molecules and interleukins. These processes were blocked by PJ34, a PARP inhibitor (32). However, in the present study, minocycline was used to inhibit the expression of PARP as opposed to PJ34.

Minocycline is a second-generation semi synthetic tetracycline and a non-specific matrix metalloproteinase blocker. Minocycline exhibits neuroprotective effects in central nervous system diseases $(12,13)$. Recently, a phase II clinical trial confirmed the therapeutic role of minocycline in the treatment of diabetic retinopathy (36). Minocycline decreases the levels of cell apoptosis through various mechanisms. The present study demonstrated that minocycline is able to inhibit PARP activation in diabetic retinopathy, which may be an important mechanism by which minocycline decreases cell apoptosis. Minocycline also exhibits powerful antioxidative effects $(37,38)$, and is able to suppress PARP activation by reducing the generation of reactive oxygen species, which are critical to PARP activation $(33,39,40)$.

In conclusion, minocycline was shown to decrease retinal cell apoptosis and increase the ERG b-wave and OP amplitudes by inhibiting abnormal PARP-1 activation in diabetic retinopathy. Since the present study was limited to examining the effects of minocycline in an in vivo diabetic rat model, further research is required in order to elucidate the mechanism underlying the inhibitory effects of minocycline on PARP-1 in diabetic retinopathy. Further research may facilitate the development of novel treatments for diabetic retinopathy.

\section{References}

1. Engerman RL and Kern TS: Retinopathy in animal models of diabetes. Diabetes Metab Rev 11: 109-120, 1995.

2. Mizutani M, Kern TS and Lorenzi M: Accelerated death of retinal microvascular cells in human and experimental diabetic retinopathy. J Clin Invest 97: 2883-2890, 1996.

3. Hammes HP, Federoff HJ and Brownlee M: Nerve growth factor prevents both neuroretinal programmed cell death and capillary pathology in experimental diabetes. Mol Med 1: 527-534, 1995.

4. Barber AJ, Lieth E, Khin SA, Antonetti DA, Buchanan AG and Gardner TW: Neural apoptosis in the retina during experimental and human diabetes. Early onset and effect of insulin. J Clin Invest 102: 783-791, 1998.

5. Szabó C and Ohshima H: DNA damage induced by peroxynitrite: Subsequent biological effects. Nitric Oxide 1: 373-385, 1997.

6. Szabó C and Dawson VL: Role of poly(ADP-ribose) synthetase in inflammation and ischaemia-reperfusion. Trends Pharmacol Sci 19: 287-298, 1998.

7. Heller B, Wang ZQ, Wagner EF, Radons J, Bürkle A, Fehsel K, Burkart $\mathrm{V}$ and Kolb H: Inactivation of the poly(ADP-ribose) polymerase gene affects oxygen radical and nitric oxide toxicity in islet cells. J Biol Chem 270: 11176-11180, 1995.

8. Li GY, Fan B and Su GF: Acute energy reduction induces caspase-dependent apoptosis and activates p53 in retinal ganglion cells (RGC-5). Exp Eye Res 89: 581-589, 2009.

9. Zheng L and Kern TS: Role of nitric oxide, superoxide, peroxynitrite and PARP in diabetic retinopathy. Front Biosci (Landmark Ed) 14: 3974-3987, 2009.

10. VirágL and SzabóC:The therapeutic potential of poly(ADP-ribose) polymerase inhibitors. Pharmacol Rev 54: 375-429, 2002. 
11. Brownlee M: Biochemistry and molecular cell biology of diabetic complications. Nature 414: 813-820, 2001.

12. Yrjänheikki J, Tikka T, Keinänen R, Goldsteins G, Chan $\mathrm{PH}$ and Koistinaho J: A tetracycline derivative, minocycline, reduces inflammation and protects against focal cerebral ischemia with a wide therapeutic window. Proc Natl Acad Sci USA 96: 13496-13500, 1999.

13. Ryu JK, Franciosi S, Sattayaprasert P, Kim SU and McLarnon JG: Minocycline inhibits neuronal death and glial activation induced by beta-amyloid peptide in rat hippocampus. Glia 48: 85-90, 2004.

14. Krady JK, Basu A, Allen CM, Xu Y, LaNoue KF, Gardner TW and Levison SW: Minocycline reduces proinflammatory cytokine expression, microglial activation, and caspase-3 activation in a rodent model of diabetic retinopathy. Diabetes 54: 1559-1565, 2005.

15. Vincent JA and Mohr S: Inhibition of caspase-1/interleukin-1beta signaling prevents degeneration of retinal capillaries in diabetes and galactosemia. Diabetes 56: 224-230, 2007.

16. Chang CJ, Cherng CH, Liou WS and Liao CL: Minocycline partially inhibits caspase-3 activation and photoreceptor degeneration after photic injury. Ophthalmic Res 37: 202-213, 2005.

17. Obrosova IG, Minchenko AG, Frank RN, Seigel GM, Zsengeller Z, Pacher P, Stevens MJ and Szabó C: Poly(ADP-ribose) polymerase inhibitors counteract diabetes- and hypoxia-induced retinal vascular endothelial growth factor overexpression. Int J Mol Med 14: 55-64, 2004.

18. Santiago AR, Cristóvão AJ, Santos PF, Carvalho CM and Ambrósio AF: High glucose induces caspase-independent cell death in retinal neural cells. Neurobiol Dis 25: 464-472, 2007.

19. Zhu B, Wang W, Gu Q and Xu X: Erythropoietin protects retinal neurons and glial cells in early-stage streptozotocin-induced diabetic rats. Exp Eye Res 86: 375-382, 2008.

20. Hotta N, Koh N, Sakakibara F, Nakamura J, Hamada Y, Naruse K, Sasaki H, Mizuno K, Matsubara A, Kakuta H, et al: Effect of an aldose reductase inhibitor, SNK-860, on deficits in the electroretinogram of diabetic rats. Exp Physiol 80: 981-989, 1995.

21. Wurziger K, Lichtenberger T and Hanitzsch R: On-bipolar cells and depolarising third-order neurons as the origin of the ERG-b-wave in the RCS rat. Vision Res 41: 1091-1101, 2001.

22. Tzekov R and Arden GB: The electroretinogram in diabetic retinopathy. Surv Ophthalmol 44: 53-60, 1999.

23. Ogden TE: The oscillatory waves of the primate electroretinogram. Vision Res 13: 1059-1074, 1973

24. Wachtmeister L and Dowling JE: The oscillatory potentials of the mudpuppy retina. Invest Ophthalmol Vis Sci 17: 1176-1188, 1978.

25. Li Q, Zemel E, Miller B and Perlman I: Early retinal damage in experimental diabetes: Electroretinographical and morphological observations. Exp Eye Res 74: 615-625, 2002.

26. Kline RP, Ripps H and Dowling JE: Generation of b-wave currents in the skate retina. Proc Natl Acad Sci USA 75: 5727-5731, 1978.

27. Klemp K, Sander B, Brockhoff PB, Vaag A, Lund-Andersen H and Larsen M: The multifocal ERG in diabetic patients without retinopathy during euglycemic clamping. Invest Ophthalmol Vis Sci 46: 2620-2626, 2005 .
28. Chen BH, Jiang DY and Tang LS: Advanced glycation end-products induce apoptosis involving the signaling pathways of oxidative stress in bovine retinal pericytes. Life Sci 79: 1040-1048, 2006.

29. Du Y, Miller CM and Kern TS: Hyperglycemia increases mitochondrial superoxide in retina and retinal cells. Free Radic Biol Med 35: 1491-1499, 2003.

30. Drel VR, Xu W, Zhang J, Kador PF, Ali TK, Shin J, Julius U, Slusher B, El-Remessy AB and Obrosova IG: Poly(ADP-ribose)polymerase inhibition counteracts cataract formation and early retinal changes in streptozotocin-diabetic rats. Invest Ophthalmol Vis Sci 50: 1778-1790, 2009

31. Obrosova IG and Julius UA: Role for poly(ADP-ribose) polymerase activation in diabetic nephropathy, neuropathy and retinopathy. Curr Vasc Pharmacol 3: 267-283, 2005.

32. Zheng L, Szabó C and Kern TS: Poly(ADP-ribose) polymerase is involved in the development of diabetic retinopathy via regulation of nuclear factor-kappaB. Diabetes 53: 2960-2967, 2004.

33. Li GY and Osborne NN: Oxidative-induced apoptosis to an immortalized ganglion cell line is caspase independent but involves the activation of poly(ADP-ribose)polymerase and apoptosis-inducing factor. Brain Res 1188: 35-43, 2008.

34. Miki K, Uehara N, Shikata N, Matsumura M and Tsubura A: Poly (ADP-ribose) polymerase inhibitor 3-aminobenzamide rescues N-methyl-N-nitrosourea-induced photoreceptor cell apoptosis in Sprague-Dawley rats through preservation of nuclear factor-kappaB activity. Exp Eye Res 84: 285-292, 2007.

35. Oku H, Goto W, Okuno T, Kobayashi T, Sugiyama T, Ota T, Yoneda S, Hara $\mathrm{H}$ and Ikeda T: Effects of poly(ADP-ribose) polymerase inhibitor on NMDA-induced retinal injury. Curr Eye Res 29: 403-411, 2004.

36. Cukras CA, Petrou P, Chew EY, Meyerle CB and Wong WT: Oral minocycline for the treatment of diabetic macular edema (DME): Results of a phase I/II clinical study. Invest Ophthalmol Vis Sci 53: 3865-3874, 2012.

37. Kraus RL, Pasieczny R, Lariosa-Willingham K, Turner MS, Jiang A and Trauger JW: Antioxidant properties of minocycline: Neuroprotection in an oxidative stress assay and direct radical-scavenging activity. J Neurochem 94: 819-827, 2005.

38. Choi SH, Lee DY, Chung ES, Hong YB, Kim SU and Jin BK: Inhibition of thrombin-induced microglial activation and NADPH oxidase by minocycline protects dopaminergic neurons in the substantia nigra in vivo. J Neurochem 95 1755-1765, 2005.

39. Sinha-Hikim I, Shen R, Nzenwa I, Gelfand R, Mahata SK and Sinha-Hikim AP: Minocycline suppresses oxidative stress and attenuates fetal cardiac myocyte apoptosis triggered by in utero cocaine exposure. Apoptosis 16: 563-573, 2011.

40. Cieslik M, Pyszko J and Strosznajder JB: Docosahexaenoic acid and tetracyclines as promising neuroprotective compounds with poly(ADP-ribose) polymerase inhibitory activities for oxidative/genotoxic stress treatment. Neurochem Int 62: 626-636, 2013. 